\title{
PERFIL EPIDEMIOLÓGICO DE PACIENTES COM LEISHMANIOSE VISCERAL
}

\author{
NOTIFICADOS EM HOSPITAL DE REFERÊNCIA EM TERESINA - PI
}

\author{
Raimundo Leoberto Torres de Sousa ${ }^{1}$ \\ Maria Ivanete Nunes ${ }^{2}$ \\ Simone Mousinho Freire ${ }^{3}$ \\ Recebido em: 23 mar. 2018 \\ Aceito em: 13 mar. 2019
}

RESUMO: Leishmaniose visceral (LV) é uma doença grave que pode apresentar letalidade de $95 \%$, é provocada pelo protozoário Leishmania infantum chagasi, transmitido pelas fêmeas de flebotomíneos do gênero Lutzomyia sp. e que afeta o homem e os animais, particularmente o cão doméstico. O objetivo deste estudo foi descrever a perfil epidemiológico de pacientes com (LV) notificados em hospital de referência de TeresinaPI, no período de 2007 a 2016. A pesquisa foi realizada no Núcleo de Vigilância Epidemiológica do hospital de estudo utilizando-se o banco de dados do Sistema de Informação de Agravos e Notificação - SINAN. Os dados foram estruturados em questionário, compreendendo as variáveis, como: idade, sexo, município de residência, escolaridade, evolução do caso e presença de outros agravos como o HIV. Os resultados apontam que há relação entre infecção por (LV) e HIV, relação com o gênero, acometendo principalmente o sexo masculino, escolaridade abordando pessoas com o ensino fundamental incompleto e idade onde os pacientes são menores de 10 anos. Os notificados em sua maioria são de Teresina. Considera-se a necessidade de programas de combate à (LV), podendo-se pontuar a necessidade de novas investigações para subsidiar tais programas no Brasil, e contribuir para compreensão da epidemiologia desta doença.

Palavras-chave: Leishmania. Zoonoses. Diagnóstico.

\section{EPIDEMIOLOGICAL PROFILE OF PATIENTS WITH VISCERAL LEISHMANIOSIS}

\section{NOTIFIED IN REFERENCE HOSPITAL IN TERESINA - PI}

ABSTRACT: Visceral leishmaniasis (LV) is a serious disease that may present lethality of $95 \%$, is caused by the protozoan Leishmania infantum chagasi, transmitted by females of sand flies of the gender Lutzomyia sp. and which affects man and animals, particularly the domestic dog. The objective of this study was to describe the epidemiological profile of patients with (LV) reported in a reference hospital in Teresina-PI in the period from 2007 to 2016 . The research was carried out at the Center for Epidemiological Surveillance of the study hospital using the database of the System of Information of Injuries and Notification - SINAN. The data were structured in a questionnaire, including variables such as: age, sex, municipality of residence, schooling, case evolution and presence of other diseases such as HIV. The results indicate that there is a relationship between (LV) infection and HIV. related to gender, affecting mainly males, schooling addressing people with incomplete primary education and age where the patients are younger than 10 years. Most of them are

\footnotetext{
1 Mestrando em medicina tropical - Fiocruz Piauí - leoberto_torres@outlook.com.

2 Especialista em Vigilância em Saúde - UESPI - mariaiva.nunes@hotmail.com.

3 Doutora em Ciência Animal - Universidade Federal do Piauí - UFPI - simoneuespi@gmail.com.
} 
from Teresina. It is considered the need of programs to combat (LV), and it is possible to point out the need for new research to subsidize such programs in Brazil, and contribute to the understanding of the epidemiology of this disease.

Keywords: Leishmania. Zoonoses. Diagnosis.

\section{INTRODUÇÃO}

A leishmaniose é causada por mais de 20 espécies de protozoários do gênero Leishmania, sendo transmitida para o homem por aproximadamente 30 diferentes espécies de flebotomíneos. Esta doença consiste de quatro síndromes clínicas principais: leishmaniose cutânea, leishmaniose visceral, leishmaniose mucocutânea e leishmaniose dermal pós-calazar (CHAPPUIS, 2007).

Deste modo podemos citar que a leishmaniose é:

[...] uma doença transmitida por vetores e causada por protozoários intra-macrófagos, é endêmica em grandes áreas dos trópicos, subtrópicos e na bacia do Mediterrâneo. Esta doença é caracterizada por diversidade e complexidade1: é causada por mais de 20 espécies de Leishmania e é transmitida aos humanos por 30 espécies diferentes de flebotomíneos. [...]

A leishmaniose visceral (LV) é uma doença protozoária sistêmica transmitida por flebotomíneos. [...] O diagnóstico e o tratamento precoces e precisos continuam sendo os principais componentes do controle da LV. (CHAPPUIS, 2007, p. 873).

A forma visceral desta doença nas Américas é causada pela Leishmania infantum chagasi, também conhecida como calazar, nome derivado da palavra hindu Kala-Aza, que significa febre negra; pois alguns doentes podem desenvolver intensa hiperpigmentação da pele, embora tal fenômeno não ocorra no Brasil. Atualmente, pode manifestar-se como doença oportunista em pacientes imunocomprometidos em decorrência da AIDS (Sindrome da Imunodeficiência Humana), na qual não são incomuns, além do envolvimento sistêmico, as lesões cutâneas (NEVES, 2011 p. 67)

A leishmaniose visceral (LV) é uma doença grave, atingindo crianças, adultos jovens ou pessoas imunodeprimidas, e quando não tratada, pode apresentar letalidade em $95 \%$ dos casos. Ela tem sido apontada como uma doença re-emergente, caracterizando nítido processo de transição epidemiológica, apresentando incidência crescente nos últimos anos nas áreas onde ocorria tradicionalmente; expandindo geograficamente para os estados mais ao sul do país, e um franco processo de urbanização em cidades localizadas em regiões distintas, como o Nordeste e o Sudeste (ALVES, 2004, p. 259).

O desenvolvimento de novas drogas e vacinas, melhores diagnósticos e o acesso dos pacientes a todos esses benefícios, são os principais desafios para o controle da LV. Portanto, o investimento em pesquisas é necessário para minimizar os problemas com doenças negligenciadas, como é o caso das leishmanioses. As características epidemiológicas e o conhecimento insuficiente sobre os elementos que compõe a cadeia de transmissão da LV fazem com que as estratégias de controle desta doença sejam pouco 
efetivas. Atualmente essas estratégias se baseiam no diagnóstico, e tratamento precoce dos doentes, redução da população de flebotomíneos, através do uso de inseticidas nas casas, eliminação dos cães soropositivos e atividades de educação em saúde (BRASIL, 2006, p. 31).

Com mortalidade global de 59.000 óbitos por ano, a leishmaniose visceral, permanece como importante problema de saúde pública em vários países do mundo. (TORRES, 2006, p. 537).

[...] No Brasil, por causa de abordagem de vários programas de controle, houve melhoras devido às definições das áreas de transmissão ou de risco; além disso, foram implementadas ações de vigilância para os municípios silenciosos, onde a doença não era notificada devido à inexistência de ações da vigilância epidemiológica nestes municípios [...] (TORRES, 2006, p. 537).

Este trabalho teve como objetivo descrever o perfil epidemiológico dos pacientes com Leishmaniose Visceral atendidos no hospital de referência em Teresina - PI, durante o período entre 2007 a 2016, através da coleta de dados epidemiológicos de pacientes acometidos por este agravo, e notificados no referido hospital.

\section{MATERIAL E MÉTODOS}

\section{TIPOS DE PESQUISA}

Trata-se de um estudo transversal, descritivo de natureza quantitativa, fundamentado em pesquisa com dados secundários dos pacientes atendidos e notificados com leishmaniose Visceral em um hospital de referência, em Teresina-PI, no período de 2007 a 2013.

\section{COLETA DE DADOS}

A pesquisa foi realizada no Núcleo de Vigilância Epidemiológica do referido hospital utilizando-se os relatórios anuais do banco de dados do Sistema de Informação de Agravos e Notificação - SINAN, com base nas fichas de notificação dos pacientes atendidos, e notificados no hospital no período de 2007 a 2016.

Como variáveis para descrever o perfil epidemiológico dos pacientes notificados com leishmaniose visceral, foram analisados aspectos diversos como sexo, faixa etária, escolaridade, local de residência, relação de infecção com os soropositivos e evolução do caso (alta ou óbito). Estes dados foram estruturados em uma ficha questionário elaboradas pelos próprios autores. 


\section{ANÁLISE ESTATÍSTICA}

Para análise estatística dos dados foi utilizado frequência simples. As informações coletadas foram digitalizadas em um banco de dados desenvolvido utilizando o software Excel da Microsoft Office 2013.

\section{RESULTADOS E DISCUSSÃO}

No período de 2007 a 2016, o hospital do estudo notificou através do SINAN, 3032 pacientes atendidos com Leishmaniose Visceral (Gráfico 1).

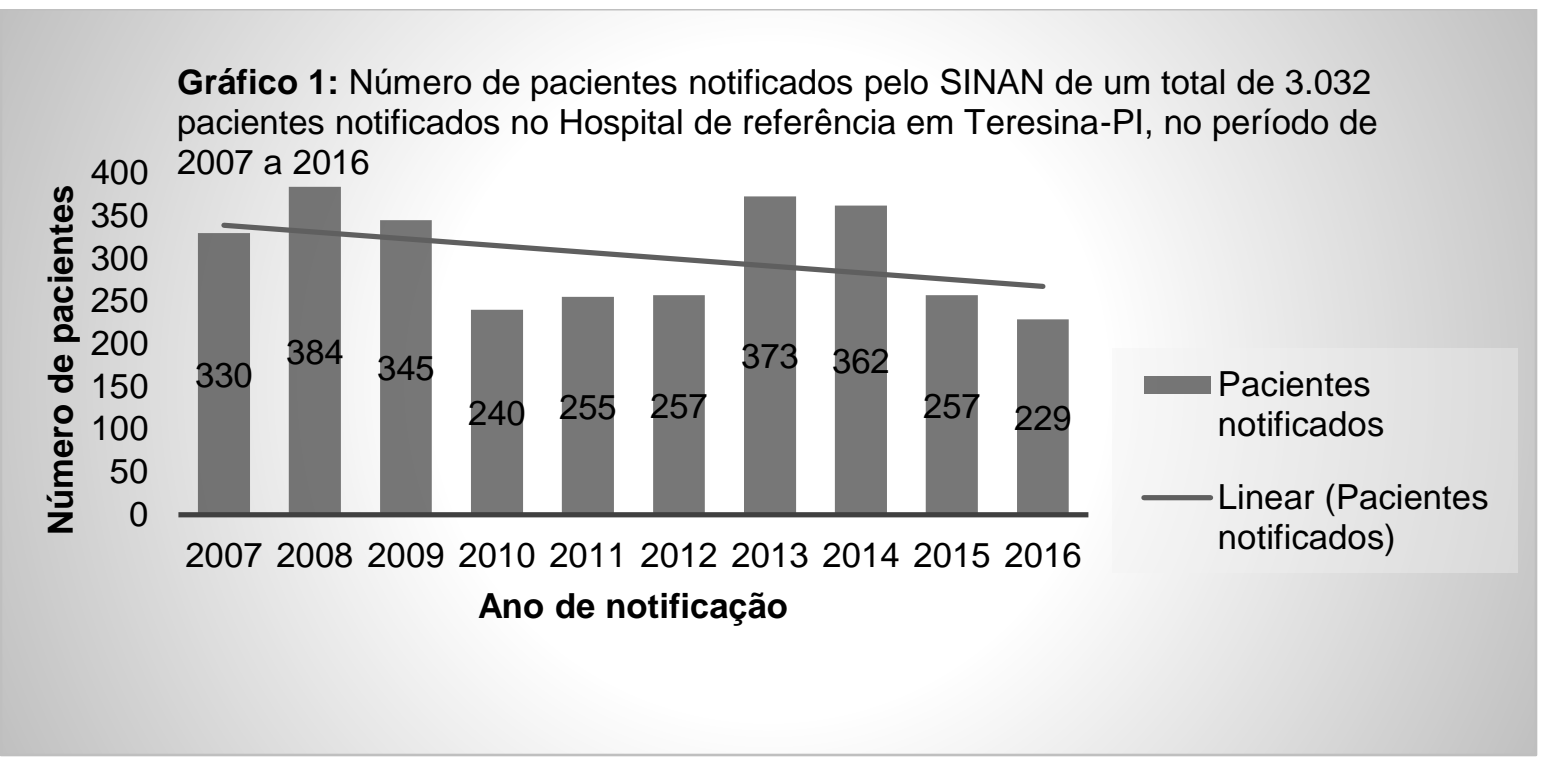

Os estados do Piauí e Maranhão são os que apresentam maior número de notificação por leishmaniose visceral. Os dados apontam que $1735(57,2 \%)$ são residentes no estado do Piauí, $1269(41,8 \%)$ no Maranhão, $20(0,65 \%)$ no Pará, $3(0,09 \%)$ no Tocantins, $3(0,09 \%)$ no Ceará, $1(0,03 \%)$ no estado da Bahia e $1(0,03 \%)$ no estado do Rio de Janeiro (Tabela 1). 
Tabela 1. Frequência de atendimento por leishmaniose visceral segundo a Unidade Federativa de residência e ano de notificação no período de 2007 a 2016.

Unidade Federativa do município de residência/Ano da notificação

\begin{tabular}{llllllllllll}
\hline \hline UF Residência & $\mathbf{2 0 0 7}$ & $\mathbf{2 0 0 8}$ & $\mathbf{2 0 0 9}$ & $\mathbf{2 0 1 0}$ & $\mathbf{2 0 1 1}$ & $\mathbf{2 0 1 2}$ & $\mathbf{2 0 1 3}$ & $\mathbf{2 0 1 4}$ & $\mathbf{2 0 1 5}$ & $\mathbf{2 0 1 6}$ & Total \\
\hline \hline Piauí & 190 & 212 & 173 & 140 & 151 & 155 & 175 & 215 & 176 & 148 & 1735 \\
\hline Maranhão & 138 & 165 & 167 & 99 & 98 & 101 & 195 & 146 & 80 & 80 & 1269 \\
\hline Pará & 00 & 06 & 04 & 00 & 06 & 01 & 01 & 01 & 01 & 00 & 20 \\
\hline Tocantins & 00 & 01 & 00 & 01 & 00 & 00 & 01 & 00 & 00 & 00 & 03 \\
\hline Ceará & 2 & 0 & 0 & 0 & 0 & 0 & 1 & 0 & 0 & 0 & 03 \\
\hline Bahia & 0 & 0 & 1 & 0 & 0 & 0 & 0 & 0 & 0 & 0 & 01 \\
\hline Rio de Janeiro & 0 & 0 & 0 & 0 & 0 & 0 & 0 & 0 & 0 & 1 & 01 \\
\hline Total & 330 & 384 & 345 & 240 & 255 & 257 & 373 & 362 & 257 & 229 & 3032 \\
\hline \hline
\end{tabular}

Fonte: SINAN

Ainda com relação à tabela 1 , observamos que no período compreendido entre 2007 e 2016, não houve redução significativa do número de notificações pelo agravo leishmaniose visceral no estado do Piauí

Dos 3032 casos de leishmaniose visceral notificados no período de 2007 a 2016, no referido hospital, $2163(71,3 \%)$ são residentes em zona urbana, $779(25,6 \%)$ na zona rural, $9(0,29 \%)$ são residentes de zonas periurbanas que compreende ambientes de transição entre zona urbana e rural e $81(2,6 \%)$ não tiveram a zona de residência identificada.

Como pode ser observado no (Gráfico 2) referente à distribuição por zonas de residência dos pacientes notificados, a maioria está representada pela zona urbana.

Gráfico 2. Distribuição dos pacientes notificados pelo SINAN de um total de 3.032 pacientes no Hospital segundo a zona de residência, no período de 2007 a 2016.

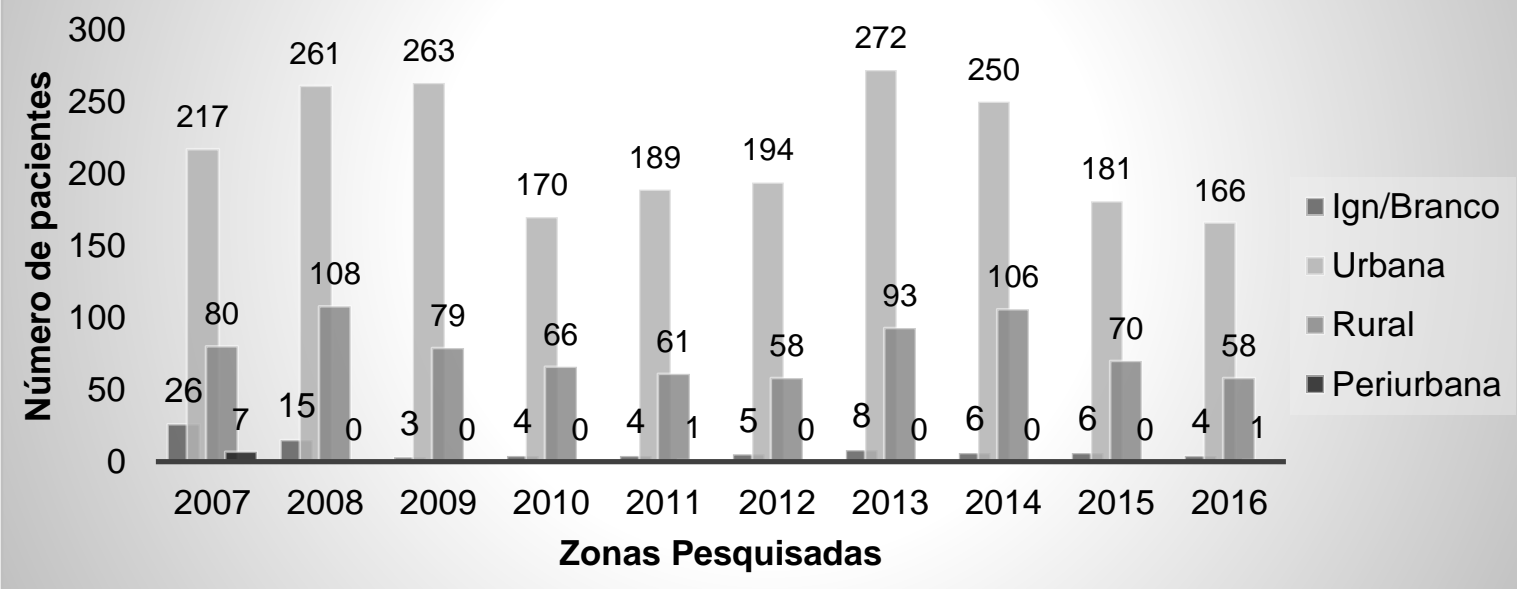

Os dados apresentados neste estudo (Gráfico 3) mostram que a maioria 2931 $(96,6 \%)$ dos casos de Leishmaniose visceral é autóctone do próprio município de 
residência, ou seja, é natural da região ou do território em que o paciente habita e foi infectado pelo vetor. Quanto aos demais foram caracterizados como não sendo autóctone do município de residência e os indeterminados por não constarem na ficha de notificação, a zona de ocorrência.

Gráfico 3. Frequência por Ano da Notificação segundo Autóctone Município de Residência dos pacientes notificados com leishmaniose visceral no período de 2007 a 2016.

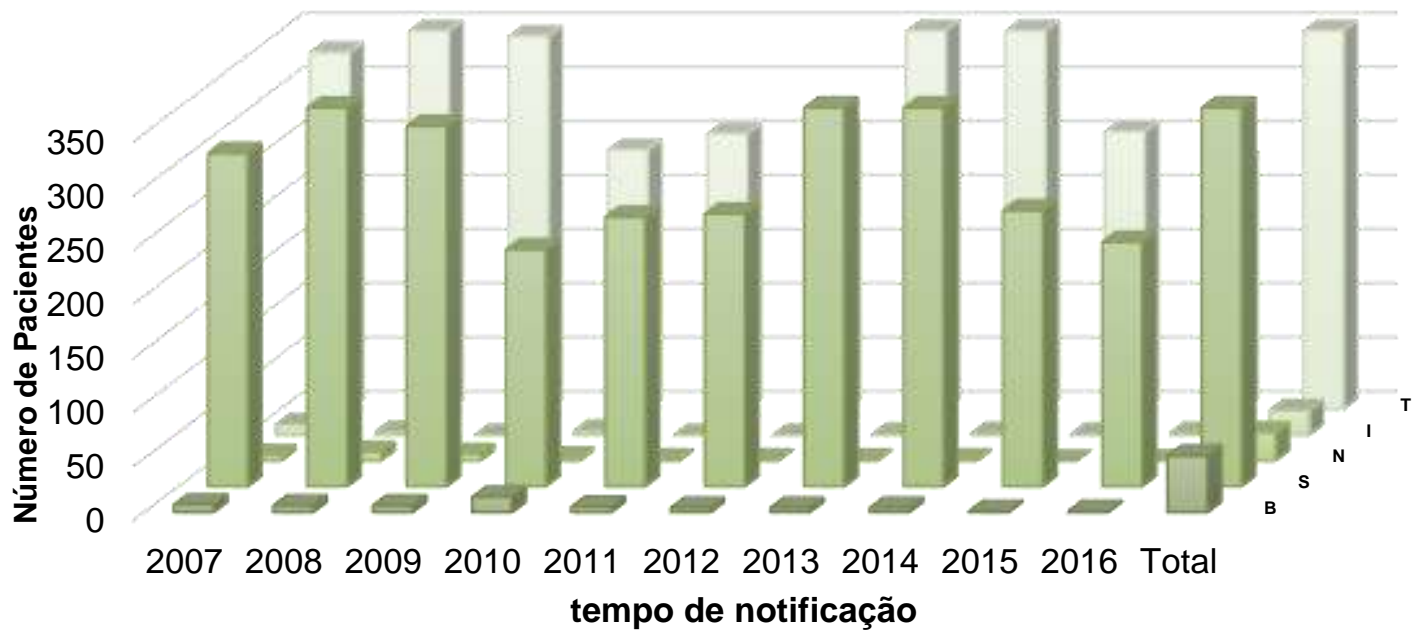

$\square B \quad \square S \quad \square N \quad \square \mathrm{N} \quad \square T$

Legenda: $\mathrm{B}=$ Branco; $\mathrm{S}=\mathrm{Sim} ; \mathrm{N}=\mathrm{Não} ; \mathrm{I}=$ Indeterminado; $\mathrm{T}=$ Total .

Em relação aos municípios com maior número de pacientes notificados com LV, destaca-se Teresina com 603 representando $34,7 \%$ do total geral de notificações por Leishmaniose visceral, seguidos pelos municípios de Miguel Alves 79 (4,5\%) e Barras 69 (3,9\%) (Tabela 2).

Tabela 2. Distribuição dos Municípios do Piauí de maior prevalência de casos de Leishmaniose visceral notificados por ano no período de 2007 a 2016.

\begin{tabular}{lllllllllllll}
\hline \multicolumn{2}{l}{ Unidade Federativa do município de residência/Ano da notificação } \\
\hline \hline Cidades & $\mathbf{2 0 0 7}$ & $\mathbf{2 0 0 8}$ & $\mathbf{2 0 0 9}$ & $\mathbf{2 0 1 0}$ & $\mathbf{2 0 1 1}$ & $\mathbf{2 0 1 2}$ & $\mathbf{2 0 1 3}$ & $\mathbf{2 0 1 4}$ & $\mathbf{2 0 1 5}$ & $\mathbf{2 0 1 6}$ & Total \\
\hline \hline Teresina & 68 & 81 & 63 & 46 & 60 & 64 & 59 & 60 & 63 & 39 & 603 \\
\hline Miguel Alves & 03 & 05 & 06 & 07 & 13 & 11 & 10 & 11 & 09 & 04 & 79 \\
\hline Floriano & 10 & 08 & 09 & 00 & 05 & 03 & 06 & 04 & 03 & 00 & 48 \\
\hline Barras & 04 & 02 & 05 & 03 & 07 & 08 & 11 & 16 & 05 & 08 & 69 \\
\hline Esperantina & 02 & 05 & 05 & 06 & 06 & 03 & 02 & 04 & 02 & 02 & 37 \\
\hline Água Branca & 03 & 00 & 00 & 04 & 03 & 01 & 03 & 02 & 00 & 01 & 17 \\
\hline José de Freitas & 09 & 03 & 02 & 03 & 01 & 03 & 05 & 03 & 04 & 08 & 41 \\
\hline Bom Jesus & 03 & 03 & 02 & 00 & 03 & 04 & 07 & 12 & 06 & 00 & 40 \\
\hline Parnaíba & 04 & 07 & 00 & 02 & 04 & 01 & 03 & 01 & 01 & 03 & 26 \\
\hline Campo Maior & 07 & 03 & 02 & 02 & 01 & 04 & 00 & 06 & 08 & 03 & 36 \\
\hline Altos & 02 & 02 & 02 & 02 & 02 & 02 & 07 & 07 & 03 & 00 & 29 \\
\hline Picos & 00 & 05 & 04 & 02 & 03 & 02 & 02 & 04 & 01 & 00 & 23 \\
\hline Piripiri & 05 & 04 & 02 & 01 & 03 & 00 & 03 & 07 & 03 & 04 & 32 \\
\hline S. R. N & 01 & 02 & 05 & 03 & 03 & 01 & 03 & 05 & 04 & 01 & 28 \\
\hline São João do Piauí & 05 & 06 & 04 & 00 & 00 & 00 & 02 & 01 & 01 & 00 & 19 \\
\hline Pedro II & 02 & 04 & 02 & 01 & 02 & 02 & 02 & 02 & 05 & 03 & 25 \\
\hline \hline
\end{tabular}

Fonte: SINAN Legenda: S. R. N = São Raimundo Nonato 
No que se refere a faixa etária, pode-se constatar que metade das pessoas notificadas com leishmaniose são menores de 10 anos de idade correspondem a 50\% (1516/ 3032) das notificações

Os dados mostram que a ocorrência por ano de notificação, segundo a co-infecção por HIV (Vírus da Imunodeficiência Humana) em pacientes com LV, no período de 2007 a 2016 foram 366 casos. Observou-se ainda que nestes pacientes há uma predominância na faixa etária de 20 a 34 anos em ordem crescente por ano de notificação correspondendo a 26,2\% (96/366) dos casos. Este resultado deve estar associado a inclusão da leishmaniose visceral como doença disseminada, na lista de infecções oportunistas.

Quanto à evolução do caso, foram identificados na pesquisa, seguindo os critérios da ficha de notificação, que 34,5\% (1048/3032) dos pacientes evoluíram para cura, 5,1\% (157/3032) para óbito por LV e 0,1\% (6/3032) para óbito por outras causas, os demais casos foram classificados como transferência, abandono e ignorados ou em branco de acordo com a ficha de notificação do paciente. Vale ressaltar que houve diminuição do número de óbitos em 2013 e 2014, comparados aos anos anteriores

Quanto à escolaridade dos pacientes, houve predominância da variável "não se aplica" que se refere a um item da ficha de notificação para caracterizar os casos ignorados quanto a escolaridade, correspondendo a 45,1\% (1322/3032) que deve estar relacionado a crianças menores de 5 anos por não ser classificada a escolaridade destas nas fichas de notificação utilizados no estudo, da $5^{\underline{a}}$ a $8^{\underline{a}}$ série do ensino fundamental corresponde a $17,7 \%$ (538/3032), seguido pelo ensino fundamental completo com 16,8\% (511/3032). No período de 2007 a 2016 foram notificados através do SINAN, 3032 pacientes com leishmaniose visceral no hospital do estudo.

Os resultados obtidos demonstram uma maior frequência desta doença no sexo masculino e em jovens menores de 10 anos de idade, confirmando a literatura segundo (ALVARENGA, 2010, p. 194)

A prevalência deste agravo ao sexo masculino pode estar correlacionada à ocorrência da LV com a implantação de projetos agropecuários, abertura de estradas e assentamento de trabalhadores rurais sem-terra, sem preocupação com infraestrutura sanitária [...] (ALVARENGA, 2010, p. 194)

Segundo (BEVILACQUA, 2001, p. 1), no ano 2000 foram registrados 3779 novos casos de leishmaniose visceral em 18 estados do Brasil. Os focos de maior endemicidade foram registrados na Bahia, Ceará, Piauí, Rio Grande do Norte e Maranhão. Isto pode ser justificado pela ineficácia ou ausência de medidas preventivas e vigilância em tempo hábil nas áreas suspeitas de presença do inseto vetor, e quanto às notificações referentes aos pacientes oriundos do Maranhão, observa-se aumento no número de notificações no hospital de estudo podendo trazer como consequência ocupação de leitos por pacientes de outros estados, tais dados coincidem com os da literatura de (COSTA, 1990 p. 361).

Segundo (FIGUEIREDO, 2014, p. 102) a Leishmaniose visceral encontra-se em processo de expansão em várias regiões brasileiras, sendo registrados casos humanos e 
caninos em áreas totalmente urbanizadas.

[...] A Leishmaniose Visceral Canina (LVC) é a zoonose mais importante, pois além da doença ser, na maioria dos casos, severa e fatal para o cão, este é ainda o reservatório da doença para os humanos. Os cães têm sido incriminados como o principal reservatório da doença, pois preenchem as condições necessárias para isso, por serem altamente susceptíveis à infecção, por possuírem elevado parasitismo cutâneo e, principalmente, devido à sua estreita relação com o homem, tanto em áreas rurais, como urbanas [...] (FIGUEIREDO, 2014, p. 102).

Aborda ainda que, duas décadas após o registro da primeira epidemia urbana em Teresina, no Piauí, o processo de urbanização se intensificou com a ocorrência de importantes epidemias em várias cidades da região nordeste, norte, centro-oeste e sudeste, o que corrobora com os resultados obtidos em nossos estudos, onde encontramos $71,3 \%$ dos pacientes oriundos da zona urbana.

Segundo (COSTA, 1990 p. 361), no estado do Piauí a LV é conhecida desde 1934 e se tornou um estado de grande importância epidemiológica no Brasil a partir de 1980, e uma endemia atingiu vasta área urbana da capital, Teresina. O resultado do trabalho nos mostra a distribuição dos pacientes notificados com Leishmaniose visceral, segundo a zona de residência. É possível observar que houve uma mudança da transmissão da Leishmania chagasi de áreas rurais para áreas urbanas que pode ser explicada pela migração de famílias pobres, que trazem consigo cães infectados, ocupação das margens de rios para fins de habitação, superpovoamento nas favelas e adaptações das famílias às condições precárias de saneamento básico, aumentando assim, a fonte alimentar dos flebotomíneos. Os dados analisados de acordo com a faixa etária do paciente apresentam resultados coincidentes com a pesquisa de (CARDOSO, 2009), que aborda sobre leishmaniose visceral no território brasileiro, e aponta que a doença atinge pessoas de todas as faixas etárias, entretanto nas áreas onde a doença é endêmica as principais vítimas, cerca de $80 \%$ dos casos registrados, são crianças menores de 10 anos. Neste estudo, as crianças menores de 10 anos correspondem à metade dos casos prevalente na pesquisa correspondendo a 50\% (1516/3032) do total de notificações.

Para (SANTANA, 2009), a razão da maior suscetibilidade da criança está ligada a maior vulnerabilidade da resposta imune, provocada pela imaturidade da imunidade humoral e celular, e pela imunodepressão induzida pela desnutrição, situação frequente nas populações pobres do Nordeste, que representa um fator de predisposição para a infecção. Segundo (MIRANDA FILHO, 2004, p. 569), entre 25 e 70\% dos adultos com leishmaniose visceral estão infectados pelo HIV, de modo que alguns autores têm defendido a sua inclusão como doença disseminada, na lista de infecções oportunistas, sugestivas do diagnóstico de AIDS.

Ainda segundo (MIRANDA FILHO, 2004, p. 569), a LV vem emergindo como doença oportunista em pessoas infectadas com o HIV, bem como em pessoas submetidas a transplantes e em associação com outras condições em que há comprometimento da imunidade mediada por células, particularmente nos portadores do HIV, situação em que a LV usualmente ocorre quando o número de células CD4+é menor que 200. 
Observou-se que há uma predominância na faixa etária de 20 a 34 anos em ordem crescente por ano de notificação correspondendo a 26,2\% (96/366) dos casos, este resultado deve estar associado a inclusão da leishmaniose visceral como doença disseminada, na lista de infecções oportunistas aos portadores de HIV.

Segundo (DANTAS-TORRES, 2006, p. 537), o índice de mortalidade por Leishmaniose visceral descreve que a doença permanece como importante problema de saúde pública em vários países do mundo. No Brasil, por causa de abordagem de vários programas de controle ele considera que houve melhoras devido às definições das áreas de transmissão ou de risco e pela implementação de ações de vigilância para os municípios silenciosos. Observa-se uma amostra significante de pacientes com baixo nível de escolaridade compreendido entre o ensino fundamental e ensino médio, estes dados podem estar relacionados ao baixo padrão sócio econômico e culturais dos pacientes acometidos pela leishmaniose visceral no período de execução da pesquisa, apenas uma pequena amostra de 7 pacientes cerca de $(0,4 \%)$ são portadores de educação superior completa.

No Brasil, de acordo com os estudos realizados, as medidas de controle da leishmaniose visceral até agora implementadas, foram incapazes para eliminar a transmissão e impedir a ocorrência de novas endemias. Fez-se necessário conhecer as variáveis para descrever o perfil epidemiológico desse grupo de pessoas acometidas por esse agravo e, a partir de então, contribuir com o desenvolvimento de políticas públicas como medida para ampliar a vigilância nas áreas de maior incidência, adoção de medidas preventivas e o diagnóstico precoce da leishmaniose visceral e suas complicações, assim como, a conscientização dos profissionais da saúde para que possa traçar protocolos de tratamentos cada vez mais direcionados às necessidades desta população visando à redução do número de internação hospitalar e índice de mortalidade pela doença.

\section{CONSIDERAÇÕES FINAIS}

Os resultados obtidos apontam que há uma relação de infecção pela Leishmaniose visceral com os soropositivos, uma relação do agravo com o sexo, escolaridade, idade e referencia que os pacientes em sua maioria são autóctones de Teresina. Considera-se a necessidade de programas de combate à leishmaniose visceral, podendo-se pontuar a necessidade de novas investigações para subsidiar tais programas no Brasil.

\section{REFERÊNCIAS}

CHAPPUIS, François., Sundar, Shyam., Hailu, Asrat., Ghalib, Hashim., Rijal, Suman., Peeling, Rosanna W., Alvar, Jorge., Boelaert, Marleen. Visceral leishmaniasis: what are the needs for diagnosis, treatment and control? Nature Reviews Microbiology, v5: p873-882, 2007. 
NEVES, D. P. Parasitologia Humana. 12 ed. Atheneu, São Paulo. p. 67, 2011.

ALVES, W. A. Reflexões sobre a qualidade do diagnóstico da leishmaniose visceral canina em inquéritos epidemiológicos: o caso da epidemia de Belo Horizonte, Minas Gerais, Brasil, 1993-1997. Cadernos de Saúde Pública, Rio de Janeiro, v. 20, n. 1, p. $259-265$, fev. 2004

BRASIL. Ministério da Saúde. Manual de vigilância e controle da leishmaniose visceral. Brasília, p 31, 2006.

DANTAS-TORRES, Filipe. Situação atual da epidemiologia da leishmaniose visceral em Pernambuco. Rev. Saúde Pública, São Paulo, v. 40, n. 3, p. 537-541, June 2006

ALVARENGA, Daniel Gomes de et al. Leishmaniose visceral: estudo retrospectivo de fatores associados à letalidade. Rev. Soc. Bras. Med. Trop., Uberaba, v. 43, n. 2, p. 194-197, Apr. 2010

BEVILACQUA, P.D. et al . Urbanização da leishmaniose visceral em Belo Horizonte. Arq. Bras. Med. Vet. Zootec., Belo Horizonte, v. 53, n. 1, p. 1-8, Feb. 2001

COSTA, Carlos Henrique N.; PEREIRA, Humberto F.; ARAUJO, Maurílio V.. Epidemia de leishmaniose visceral no Estado do Piauí, Brasil, 1980-1986. Rev. Saúde Pública, São Paulo, v. 24, n. 5, p. 361-372, Oct. 1990

FIGUEIREDO, M. J. de F, M, de; SOUZA, N. F. de; FIGUEIREDO, H. F. de; et al Fatores de Risco e Classificação Clínica Associados À Soropositividade para Leishmaniose Visceral Canina, Revista Ciência Animal Brasileira, v. 15, n. 1; p. 102-106, 2014

CARDOSO, J F. Estratégias para o Diagnóstico da Leishmaniose Visceral Canina em Ações de Vigilância. 2009 36f Dissertação (Mestrado em Biologia de Agentes Infecciosos e Parasitários) Universidade Federal do Pará, 2009.

SANTANA J. S, SILVA A. R, CAVALCANTE M. N. S, et al. Condições socioeconômicas, estado nutricional e consumo alimentar de crianças com Leishmaniose visceral atendidas em serviço público de saúde da cidade de São Luís, Maranhão, Brasil.Caderno de Pesquisa. v. 16, n. 22009

MIRANDA FILHO, D. B. Leishmaniose visceral e tegumentar. In: MELO, H. R. et al. Condutas em doenças infecciosas. Rio de Janeiro: Medsi,;:. Cap. 51. p. 569-577, 2004 